\title{
Aspectos interventores en la participación política y electoral de jóvenes. Una reflexión sobre la información, interacción y difusión de contenidos en redes sociales para futuras investigaciones en Santander
}

CArlos Alberto Durán SÁNCHEZ*

Artículo recibido: 2 de junio de 2014

Artículo aprobado: 18 de diciembre de 2014

Doi: dx.doi.org/10.12804/desafios27.01.2015.02

Para citar este artículo: Durán, S. C. A. (2015). Aspectos interventores en la participación política y electoral de jóvenes. Una reflexión sobre la información, interacción y difusión de contenidos en redes sociales para futuras investigaciones en Santander. Desafíos 27(1), 47-81. doi: dx.doi.org/10.12804/desafios27.01.2015.02

\section{Resumen}

La participación política juvenil es uno de los aspectos notorios en las redes sociales que, sin embargo, no se ve reflejada en un aumento de la participación electoral, toda ver. que los niveles de abstención continúan siendo grandes en Colombia. Existen varias condiciones que merecen ser tenidas en cuenta para futuras investigaciones sobre el tema, que van desde los enfoques de la decisión racional y el socio-sicológico, pasando

* Profesor Investigador. Universitaria de Investigación y Desarrollo (Udi), Facultad de Comunicación Social, Bucaramanga, Colombia. Correo electrónico: carlosadurans@ yahoo.es 
por las características que posee la información transmitida y el comportamiento entre lazos fuertes o débiles de los usuarios, hasta las prácticas deliberativas más usadas como los memes y la construcción de la auto imagen. Este articulo es un intento por recopilar algunos de estos aspectos, los cuales podrían ser abordados desde lo individual o lo colectivo, ya que estos espacios políticos virtuales son escenarios constitutivos de nuevos contextos sociales que ya no pueden ser desconocidos, ni por candidatos ni por ciudadanos. Analizar los aspectos que podrían intervenir tanto en la movilización como en la identificación partidista, constituye un recurso para observar los nuevos vinculos entre la democracia, la ciudadanía, y los sistemas de información.

Palabras clave: Elecciones, redes sociales, jóvenes, información, nuevos contextos políticos, participación política.

\title{
Aspects Involved in the Political and Electoral Participation of Young People. A Reflection About Information, Interaction, and Dissemination of Contents on Social Networks for Future Research in Santander
}

\begin{abstract}
Youth political participation is a notorious aspect in social networks that, however, does not translate into an increase in voter turnout since electoral abstention levels in Colombia remain high. There are several aspects that deserve consideration for future research on the subject, ranging from rational decision and socio-psychological approaches, to the features of the information being transmitted and the behavioral differences between users with strong and weak ties, and finally more deliberative practices being used such as Memes and self-image building. This article is an attempt to compile some of these issues, which could be addressed from an individual or collective perspective, as these virtual spaces are constitutive political scenarios of new social contexts that cannot be ignored, either by candidates or citizens. Analyzing the aspects that could intervene in both mobilization and party identification constitutes a resource in order to observe the new emergent links between democracy, citizenship, and information systems.
\end{abstract}

Keywords: Elections, social networks, youth, information, new political contexts, political participation. 


\title{
Aspectos interventores na participação política e eleitoral de jovens. Uma reflexão sobre a informação, interação e difusão de conteúdos em redes sociais para futuras pesquisas em Santander
}

\begin{abstract}
Resumo
A participação política juvenil é um dos aspectos notórios nas redes sociais que, contudo, não se reflete em um aumento da participação eleitoral, toda vez que os niveis de abstenção continuam sendo grandes na Colômbia. Existem várias condições que merecem ser tidas em conta para futuras pesquisas sobre o tema que vão desde os enfoques da Decisão Racional e o Sócio psicológico, passando pelas características que possui a informação transmitida, e o comportamento entre laços fortes ou fracos dos usuários, até as práticas deliberativas mais usadas como os Memes e a construção da autoimagem. Este artigo é uma tentativa por recopilar alguns destes aspectos, os quais poderiam ser abordados desde o individual e o coletivo, devido a que não podem ser desconbecidos, nem por candidatos nem por cidadãos. Analisar os aspetos que poderiam intervir tanto na mobilização quanto na identificação partidarista, constitui um recurso para observar os novos vinculos entre a democracia, a cidadania, e os sistemas de informação.
\end{abstract}

Palavras-chave: Eleições, redes sociais, jovens, informação, novos contextos políticos, participação política.

\section{Introducción}

La información tiene en la postmodernidad una particularidad especial que acrecienta su característica de poder, la de contar con instrumentos digitales novedosos para ser difundida de manera rápida, participativa —-muchas veces de manera anónima—, lo que ayuda en la construcción de estereotipos sociales y contribuye en el cambio de actitudes humanas. Las redes sociales, Facebook y Twitter, son un espacio visible en el que interactúan ciudadanos con enormes diferencias de valores y con vínculos y afinidades que se entrelazan, se aceptan o se rechazan. Para la actividad política, son diversas las 
variables que se pueden observar en los muros virtuales, conservándose en lo demográfico un factor interesante, toda vez que son los jóvenes quienes hacen uso de estos medios con mayor intensidad. Se encuentra que la población joven tiene como característica especial ser parte activa y participativa de la esfera pública, sin embargo, en Colombia se mantiene alejada de algunos procesos de participación política tales como la inclusión en partidos políticos o las elecciones.

La participación en redes sociales tiene como característica, además de la movilidad, acrecentada por la posibilidad de hacer uso de teléfonos móviles y tablets con plataformas tecnológicas rápidas, la del manejo de una autonomía en la interacción, de acuerdo con múltiples factores que varían según los contextos socioculturales. Este concepto autónomo tiene implicaciones en lo sociopolítico. Según Castells (2008), lo que está detrás de la participación creciente, además de una crisis de legitimidad de los partidos políticos y líderes, también es la pérdida de credibilidad en los medios de comunicación que son vistos como alienados servilmente a los dirigentes; en consecuencia, los ciudadanos han requerido tomar los asuntos en sus propias manos utilizando "momentos de indignación para establecer la política insurgente como un nuevo elemento en el sistema político" (p. 449).

El presente artículo pretende proporcionar algunos elementos para futuros análisis acerca de la participación política y electoral de los jóvenes que hacen uso de las redes sociales y que además reúnen componentes desde el enfoque de la decisión racional, tales como la decisión de conseguir empleo, la incomprensión, el descontento o la desidia hacia los temas políticos, los candidatos o el sistema democrático. Igualmente, desde el enfoque sociológico es latente la necesidad de vincularse socialmente por medio de redes integradoras que motivan la auto confianza y que se desarrollan a medida que el individuo avanza cronológicamente en edad. La reflexión sobre los temas electorales tiene que ver precisamente con abrir preguntas que pudieran ser especificadas para el movimiento de las redes sociales en la ciudad de Bucaramanga, especialmente Facebook y su vínculo con la participación electoral real. 
Luego de una reflexión sobre tres grandes factores que destacan la importancia de hacer estos estudios, este artículo pretende sintetizar en las conclusiones los aspectos que pueden servir como puntos de mira para futuras investigaciones, específicamente proyectadas para Bucaramanga y los municipios que comprenden su área metropolitana.

\section{Factor edad y participación política en Colombia}

El comportamiento electoral tiene correspondencia explicativa con factores que lo afectan a largo o a corto plazo. Dentro de los enfoques, que en teoría política se encuentran vigentes, está el de la decisión racional, que concentra la práctica democrática y la decisión del votante como el resultado de un análisis acerca de los beneficios que obtiene el ciudadano a corto plazo, tanto económicos como de las opiniones sobre las campañas, los candidatos y el análisis público de las gestiones gubernamentales. Sin embargo, quizá el enfoque que podría dar mayor explicación a este comportamiento es el sociopsicológico, especialmente cuando expone que una de las causas por la cual los jóvenes votan menos es porque su identificación partidista está menos consolidada que en los adultos, ya que esta se afianza con su reiterada vinculación a la participación política y al ejercicio de votar en reiteradas elecciones. Por ello, se trata de un "Concepto alternativo de Fiorina y el enfoque de decisión racional: Un mecanismo de decisión (guía) sometido a evaluación política continua" (Molina, 2014, p. 2).

El factor edad no sería reconocido como un clivaje, aunque en una primera instancia podría pensarse que lo es porque divide la sociedad en dos bandos (jóvenes y viejos); se trata de una variable estructural, ya que el individuo pasaría en su recorrido vital, desde que vota hasta que muere, por todas las categorías. Es decir, no se mantiene anclado, porque si lo estuviera, todos formarían parte en algún momento de las filas de oposiciones políticas. Ahora bien, según el trabajo de Anduiza y Boch (2004), es necesario agrupar las diferencias de voto según la edad, de manera regular en los tres grandes comportamientos que son su consecuencia: los jóvenes votan más a partidos nuevos, los jóvenes votan más a partidos más radicales y los jóvenes responden 
más a la atmósfera del momento, es decir, generalmente a factores de corto plazo. No obstante, como característica social y demográfica hay que tener en cuenta la edad, pues esta incide en las elecciones.

En términos generales, la población joven en Colombia, con edades comprendidas entre los 18 y los 29 años, podría decidir en forma significativa los resultados, ya que conservan el $46 \%$ del potencial electoral, según un estudio de ICP Colombia (Clavijo \& Morera, 2010) constituye un factor estadísticamente definitorio para cualquier candidato.

La proyección de población en Colombia para el año 2014 está estimada en 47121089 y el censo de edades comprendidas entre los 18 y los 26 años en 7618593. La población de Santander sería de 324828 y la del Área Metropolitana de Bucaramanga (que incluyen a los municipios de Floridablanca, Piedecuesta y Girón) de 173226. De estos jóvenes, los de edades comprendidas entre los 18 y los 19 años, quienes votarían por primera vez (primivotantes) en el año 2014 corresponden a 38848 ciudadanos en el área metropolitana, este dato se construyó con base en los cuadros proyectivos de población de los archivos del Departamento Nacional de Estadística (DANE).

El caso colombiano conserva las tendencias generales de los votantes, en primer lugar de corresponder a un grupo notoriamente poco participativo, especialmente los llamados primivotantes y, en segundo lugar, del aumento de la participación electoral a medida que la edad aumenta.

Tabla 1. Ejemplo participación electoral juvenil. Elecciones para autoridades locales 2003, Colombia

\begin{tabular}{|c|r|r|r|r|r|r|}
\hline \multirow{2}{*}{ Edad } & \multicolumn{2}{|c|}{ Femenino } & \multicolumn{2}{c|}{ Masculino } & \multicolumn{2}{c|}{ Total } \\
\cline { 2 - 7 } & Número & $\%$ & Número & $\%$ & Número & $\%$ \\
\hline 18 & 50690 & 3,1 & 50759 & 4,1 & 101449 & 3,5 \\
\hline 19 & 161589 & 10 & 143176 & 11,5 & 304765 & 10,7 \\
\hline 20 & 293101 & 18,2 & 168370 & 13,5 & 461471 & 16,1 \\
\hline 21 & 207545 & 12,9 & 165813 & 13,3 & 373358 & 13,1 \\
\hline
\end{tabular}




\begin{tabular}{|c|c|c|c|c|c|c|}
\hline \multirow{2}{*}{ Edad } & \multicolumn{2}{|c|}{ Femenino } & \multicolumn{2}{c|}{ Masculino } & \multicolumn{2}{c|}{ Total } \\
\cline { 2 - 7 } & Número & $\%$ & Número & $\%$ & Número & $\%$ \\
\hline 22 & 168030 & 10,4 & 144505 & 11,6 & 312535 & 10,9 \\
\hline 23 & 155189 & 9,6 & 126112 & 10,1 & 281301 & 9,8 \\
\hline 24 & 231430 & 14,4 & 175070 & 14 & 406500 & 14,2 \\
\hline 25 & 188525 & 11,7 & 148937 & 11,9 & 337462 & 11,8 \\
\hline 26 & 155710 & 9,7 & 126248 & 10,1 & 281958 & 9,9 \\
\hline Total & 1611809 & 100 & 1248990 & 100 & $\mathbf{2 8 6 0 7 9 9}$ & $\mathbf{1 0 0}$ \\
\hline
\end{tabular}

Fuente: Presidencia de la República de Colombia. Programa presidencial Colombia Joven (2004)

Las elecciones del año 2003, convocadas para autoridades locales (Alcalde, Gobernador, Asambleas y Concejos), tuvieron un censo electoral de 25069773 y una participación para gobernadores total de 10469685 votos, la participación de jóvenes en edades comprendidas entre 18 y 26 años fue de 2860799 , es decir de un $27,32 \%$.

Sin embargo, el abstencionismo con referencia al potencial electoral de los jóvenes en Colombia sigue siendo alto. El Registrador Nacional del Estado Civil, Carlos Ariel Sánchez, exponía que las razones por las cuales solo un 10\% del potencial electoral de los jóvenes en edades comprendidas entre los 18 y los 24 años vota, una cifra baja relacionada con el $50 \%$ de los mayores de 40 años, podrían deberse "a factores de conocimiento de una parte, y de otra, [a] su grado de confianza en las instituciones. La gente joven, generalmente es rebelde y contestataria, y puede expresar la inconformidad con la abstención” (Sánchez, 2013).

Un factor que serviría para explicar la baja participación electoral en relación con la edad tiene que ver con elementos de aprendizaje y con la consecución de recursos económicos que, según Anduiza y Bosch (2004), tienden a adquirirse con el tiempo. Otros aspectos que podrían explicar las razones por las cuales los jóvenes votan menos y los mayores más serían, en primer lugar, las marcadas diferencias generacionales que hacen que los jóvenes colombianos se identifiquen con grupos de edad y con contextos políticos diferentes a los de sus 
padres y abuelos. Por ejemplo, la generación de los años ochenta, época de narcotráfico, y la del noventa, época de crisis política y de violencia, es diferente a la generación de ciudadanos que ven una mayor prosperidad económica en la segunda década del siglo XXI, o de quienes visualizan un posible posconflicto y una relativa paz. Esto provoca diferentes niveles motivacionales de movilización hacia las urnas, especialmente si se encuentra que hay una generación marcada por ser más participativa que otra.

En segundo lugar, tiene que ver con el nivel de consecución de recursos económicos, que puede ir variando desde los jóvenes primivotantes, pasando por los universitarios y nuevos profesionales que buscan empleo por primera vez, hasta quienes están afianzados con una mayor estabilidad económica y mejores ingresos. Esto se debe a que un mayor salario podría significar que la energía dedicada a la decisión de votar estuviera motivada por la reflexión y el análisis, más que por la necesidad económica del momento. En Colombia, son muchos los jóvenes que trabajan en campañas políticas, encargándose principalmente de repartir publicidad, o en labores logísticas y se sabe que votan con la perspectiva de que su candidato aprovisione empleo, con la esperanza de verse constituidos dentro de una red clientelar. No obstante, cuando son jóvenes estudiantes y aún no tienen la necesidad económica de mantener una familia, podrían verse abocados a la abstención.

En tercer lugar, el aprendizaje y conocimiento sobre asuntos políticos podría estar relacionado con un mayor o menor nivel de estudio y también con un mayor o menor nivel de información. En el primer caso, podría deberse a las destrezas proporcionadas por un mayor horizonte educativo sobre la política, la democracia y las habilidades para hablar en público, debatir y opinar de manera participativa. Al tener un mayor nivel de conocimiento de carácter científico sobre la política y sobre los asuntos que atañen al país, podría verse estimulada la participación electoral y una mayor integración social. En el segundo caso, la información sobre política, es decir, el conocimiento que necesariamente no tiene carácter científico, estimulado principalmente por el acceso a nuevas tecnologías de información y 
comunicación como las redes sociales y los blogs, también podrían motivar un comportamiento electoral participativo.

Según análisis realizados sobre el comportamiento electoral de los adultos mayores durante las elecciones españolas 1993-2008 (Rodríguez, 2013), se observa igualmente esta baja participación de los jóvenes de 18 a 24 años con una media de índice de voto del 76,8\% frente al 91,66\% de los ciudadanos de 55 a 64 años.

Dentro de los enfoques teóricos metodológicos que en la Ciencia Política explican la participación electoral está el sociológico, cuya premisa básica es que el comportamiento electoral está fuertemente marcado por la definición propia y grupal de lo que el ciudadano cree que es en la sociedad y la manera en que deriva su comportamiento, aportando finamente las condiciones para explicar la abstención, teniendo la edad como uno de sus elementos importantes. Sin embargo, Milbrath y Goel (citados por Pérez, 2006) afirman que "no es la edad cronológica lo que importa sino la experiencia y la adquisición de responsabilidades que se relacionan con la edad" (p. 79).

El tema de los múltiples entornos que van cambiando con el tiempo, criterio descrito por Verba, Schlozman y Brady (Citados por Scherman, Arriagada \& Valenzuela, 2011) dependen también de las habilidades y recursos adquiridos que podrían presentarse y que, en el caso de las nuevas tecnologías, podría presumirse como de mayor destreza en los jóvenes.

\section{Las nuevas tecnologías como medio de información y la participación política juvenil}

Además de la edad como factor importante en las elecciones en Colombia, también podría existir una correlación con el uso de nuevas tecnologías y el acceso a la información como variables que inciden o podrían incidir en la conducta electoral. Existen numerosos análisis sobre la información, la opinión pública y el comportamiento del votante que dan cuenta de que la mayoría de ciudadanos generalmente mantienen información inexacta sobre los asuntos políticos, mientras 
hay un electorado altamente informado que es minoría. "Hay algunos cuantos que saben mucho de política y otros muchos que saben poco o saben nada acerca de ella" (Pérez, 2008, p. 5). Más allá de los enfoques que explicarían si los jóvenes votan más de manera racional o de manera emocional, se quiere central el interés en la información que incidiría en esta decisión.

El flujo de la información, acerca de las situaciones políticas y de los candidatos, que va a los hogares depende de un proceso de tamizado de los medios de comunicación que, según Giovanni Sartori (citado por Berganza \& García, 2005), no lleva a comprender las cosas sino que simplemente da nociones, presentándose una sub información, es decir, aquellas informaciones que, aunque objetivamente importantes porque constituyen opiniones públicas sobre temas públicos, son reducidas en exceso. "Numerosas informaciones son solo frívolas sobre sucesos sin importancia o tienen un simple y puro valor espectacular. Lo que equivale a decir que están desprovistas de valor o relevancia 'significativa"' (p. 22). Igualmente, también existe la información deformada, a veces con un propósito político, es decir, hay desinformación.

Sin embargo, no por existir una menor información o conocimiento podría desestimarse una elección racional. Esto se explica ya que, si existe una identificación partidista, preferencias o animadversión hacia los candidatos o partidos, el votante es capaz de guiarse de manera racional por "pequeñas y breves piezas de información acerca de la política" (Pérez, 2008, p. 5), que constituyen mensajes simples y que llevan a tomar decisiones electorales de manera razonada.

Los medios difusores de información tradicionales (prensa, radio y televisión) han ido perdiendo espacio, en cuanto a la inmediatez y la rapidez en la transmisión, con la irrupción de las redes sociales como medio de interacción en el activismo político. El uso de las tres redes sociales actualmente más populares para compartir información, Facebook, Twitter y Youtube, posibilita que los ciudadanos con motivaciones políticas presenten contenidos muy interesantes, toda 
vez que la información expresada y compartida tenga características de defectibilidad y trascendencia que antes no se conocían.

Un ejemplo cercano y exitoso fue el de las campañas presidenciales de Barack Obama en Estados Unidos, que, según el informe How Obama Really Did it (David, 2008), tuvo como eje central para ganar popularidad, compartir contenido en redes sociales como Facebook y MySpace, pero además posicionarse como un microblogger ${ }^{1}$ generador de contenido con muchos seguidores y con la capacidad de propagar viralmente mensajes de texto con contenido de multimedia ligado a YouTube (Por ejemplo, su discurso sobre la raza fue visto más de 4 millones de veces) y las convocatorias por medio de su famosa página de Internet (conocida con las siglas de Mybo) lograron incluso organizar fiestas simultáneas en las casas de algunos simpatizantes en todo el país durante su campaña del 2008.

Las formas tradicionales de participación política se han ido minimizando con respecto a la intervención de las nuevas tecnologías. Los jóvenes se sienten abanderados de estos nuevos movimientos, por ejemplo, los datos generales sobre el uso de redes sociales en Colombia indican que el grupo que más utiliza Facebook está comprendido en el rango de edad de los 18 a 24 años, con 6234020 usuarios activos, mientras que Twitter cuenta con 1378353 usuarios activos en ese mismo rango de edad (Meridean Group, 2012).

En cuanto al uso de Twitter en Santander, según datos estadísticos de Meridean Group, quienes hicieron una muestra cuantitativa de 259620 autores durante una semana, que generaron 920968 publicaciones (tweets), solo el 4,69\% correspondió al Departamento de Santander, (sin embargo, es el cuarto departamento por debajo de Cundinamarca, 20\%, Valle del Cauca, 13,39\%, y Antioquia, 11,76\%).

\footnotetext{
1 Aunque las características iniciales de los microbloggers en sus inicios, especialmente Twitter (2006-2007) como sistema de mensajería instantánea compartida era, la de ser únicamente de texto, fue evolucionando de tal manera que ya se pueden compartir imágenes, videos y mensajes cortos de voz.
} 
En esta muestra, los mensajes que tienen que ver con política fueron establecidos así:

Figura 1. Principales temas de los que hablan los colombianos en Twitter

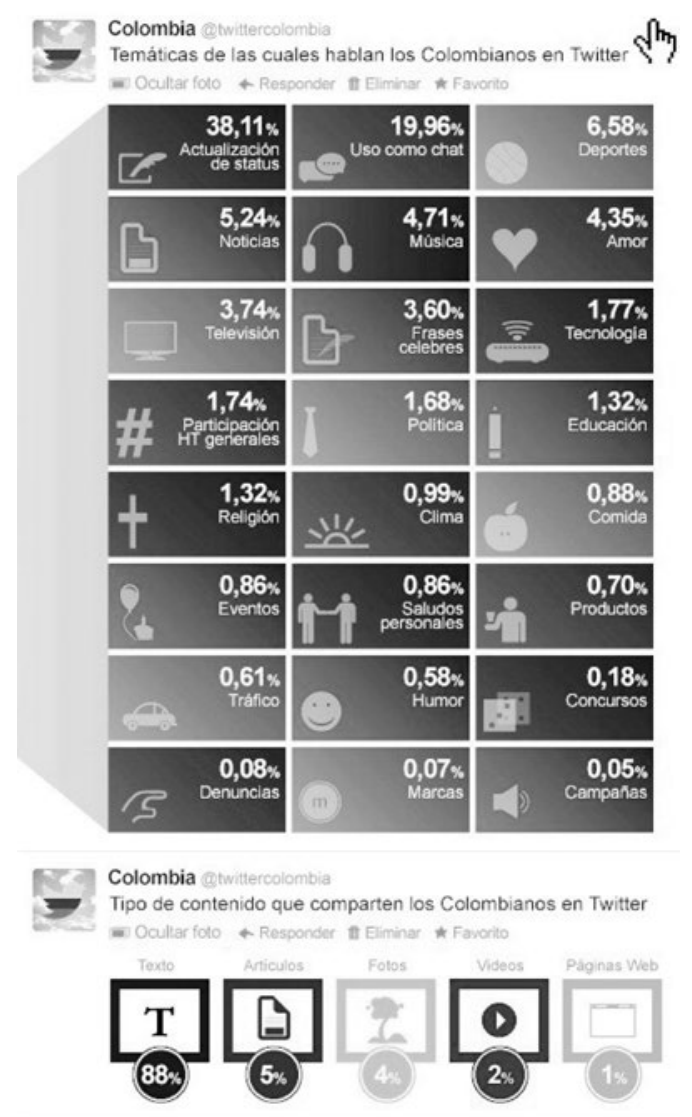

Fuente: Meridean Group (2014)

Nota: Muestra tomada del 25 al 31 de Abril de 2012. Muestra cuantitativa: 920768 tweets, 259620 autores.

En la red social Twiter, los mensajes políticos (1,68\%) corresponden principalmente a textos $(88 \%)$.

En cuanto a la participación, si bien es cierto que el desinterés de los jóvenes como sujetos políticos es muy alto en América Latina, también lo es su potencial. Por ejemplo, en Panamá los jóvenes entre los 18 y los 25 años tienen el $20 \%$ del potencial electoral y el 
abstencionismo juvenil, que era del 27,1 \% en 1994, aumentó al 32,7\% en el año 2009. La participación política en ese país en Twitter va en aumento, auspiciado por el grupo "Somos ciudadanos no clientes" de la Universidad Santa María La Antigua (USMA), quienes son conscientes de su poder electoral como grupo y proponen la reducción del abstencionismo. Este deseo es señalado por algunos analistas, ante la necesidad de atraer a la juventud, ya que los estudios indican una marcada apatía explicada por causas "que van desde el desencanto o desconfianza con el sistema o los partidos políticos, demanda por ser escuchados y tomados en cuenta, hasta la apertura de canales para una participación más activa en la política” (Bravo, 2013, p. 1). También constituyen variables que tienen concordancia con la necesidad de conseguir empleo como un factor a corto plazo, especialmente cuando se asocia a la corrupción política, al clientelismo y al desinterés estatal.

Un reciente análisis sobre la percepción política en Bucaramanga, realizado por Álvarez, Granados y Hernández (2013), encontró que al correlacionar variables existe, en los jóvenes universitarios en la ciudad de Bucaramanga, una actitud desfavorable hacia la democracia y poca comprensión política porque encuentran que la información es confusa, sin embargo, también expresan un alto optimismo hacia la democracia y una alta valoración de los líderes, los partidos, la participación electoral y la eficacia de la política. Por otro lado, la validez de sus conceptos podría generar limitaciones porque los encuestados reconocen que tienen poca información.

También es importante tener en cuenta que las informaciones sobre la política y los asuntos públicos podría estar sesgada o no ser objetiva. Almazán (citado por Sojo, 2011) subraya que el proceso de construcción de opinión no está edificado sobre la verdad sino sobre versiones de ella reducidas: "la opinión pública se convierte en un problema reducido a las formas de medición que resumen el proceso en la sumatoria de las opiniones de un colectivo" (p. 34).

En el trabajo de Álvarez et al. (2013) también vale la pena resaltar que la edad guarda una correlación directa con las actitudes políticas y, aunque débil, es posible observar que a mayor edad, mayor 
satisfacción por la democracia, pero también, a mayor edad, la actitud hacia la política es menor. En nuestro parecer, las ambigüedades e indefiniciones de parte de los estudiantes encuestados corresponde también a un nivel de desinformación sobre los temas.

La participación electoral es un concepto que hace parte de la participación política, en el que se incluyen mecanismos menos tradicionales como la protesta, el consumo, la transmisión de información política y la caricaturización de candidatos, partidos y episodios. Por eso, como señala Anduiza, Cantijoch, Colombo, Gallego y Salcedo (2010), la frontera entre la comunicación de los nuevos medios como Facebook, Twitter y YouTube y la llamada participación política se ve difuminada y cada vez menos entendida.

De hecho, las actividades políticas en estas redes son extendidas cuando ciertos tópicos políticos se vuelven populares. Por ejemplo, las críticas a las actuaciones del Congreso colombiano, las campañas de solidaridad contra el maltrato animal, la defensa del medio ambiente, o las pasiones suscitadas a favor o en contra del expresidente Álvaro Uribe Vélez. Es decir, si se trata de participación política, habría que medir la efectividad de la interacción juvenil con estos temas y si ellos se materializan como participación electoral.

Sin embargo, hay un factor que merece ser tenido en cuenta: quienes más comparten contenido en Facebook y Twitter en Colombia son los mismos medios tradicionales (radio, prensa y televisión), que han migrado a estas nuevas expresiones informativas.

\section{Tabla 2. Uso de Twitter por los medios de comunicación} e información política

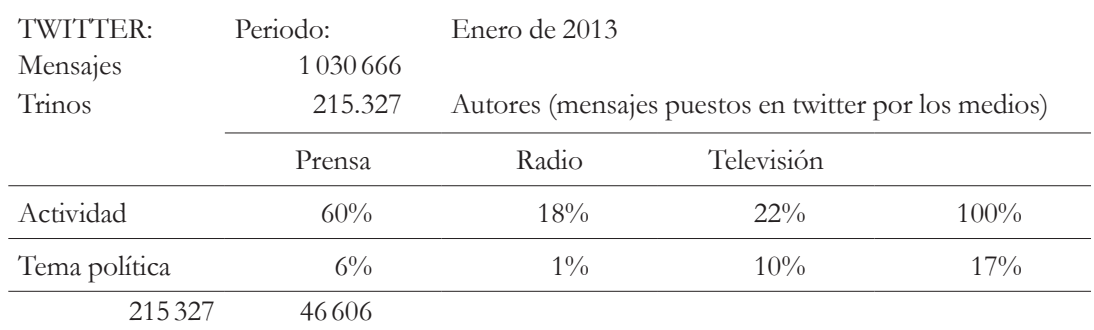

Fuente: Meridean group (2014) 
Se podría decir que de 215327 mensajes o trinos puestos por los medios de comunicación, 36606 correspondieron a temas políticos. Aunque no está analizada la interacción, es decir, si quienes vieron esos trinos en su pantalla reaccionaron profundizando en el tema al hacer enlace con el contenido en el sitio original, sí puede darnos un panorama de que el tema político conserva una franja relevante en el uso de estas redes sociales.

Precisamente, la importancia de su uso y de Internet como medio para anunciarse y de interactuar entre actores políticos ya había sido visualizada tempranamente por Castells (2000) como una nueva ágora política, que al futuro habría de incidir en una mayor ciudadanía. Se considera importante dicha participación, mirando si la generación de contenidos por parte de candidatos, la interacción y la opinión del electorado joven (18-24 años) tiene varias incidencias. La interacción en el medio es correlacional a la votación real, y si en ello tiene que ver un nivel de información adecuado o no en la toma de decisiones.

Según una reciente encuesta del Movimiento de Observación Electoral en Colombia, son los jóvenes quienes tienen mayor tendencia a votar influenciados por los medios, y los temas que son mayormente decisivos en la elección son las propuestas de los candidatos (87,6\%), la trayectoria académica y laboral $(86 \%)$ y, en tercer lugar, la información que divulgan los medios de comunicación (69,9\%), factores que podrían ser relacionados en un medio como Facebook y Twitter. Sin embargo, otro contenido comúnmente compartido en las redes, la opinión de amigos y conocidos, mantiene un rango menor (46,1\%).

A la alta importancia que para los electores tiene la información suministrada por los medios de comunicación, compartida en un alto porcentaje en redes sociales (por ejemplo un $17 \%$ en Twitter), junto a la opinión de comentaristas y columnistas, también se le suma la opinión de amigos y conocidos, igualmente compartida en un alto grado. De hecho, el incremento de ciudadanos con cuentas en Facebook, con respecto al número de habitantes en Colombia, conserva características muy interesantes, ya que de una población estimada de 47800000 colombianos, existen aproximadamente 22000000 de 
Figura 2. Percepción sobre los medios de comunicación al escoger candidato por rango de edad

¿Qué tan influyente son para usted los medios de comunicación a la hora de escoger un candidato?

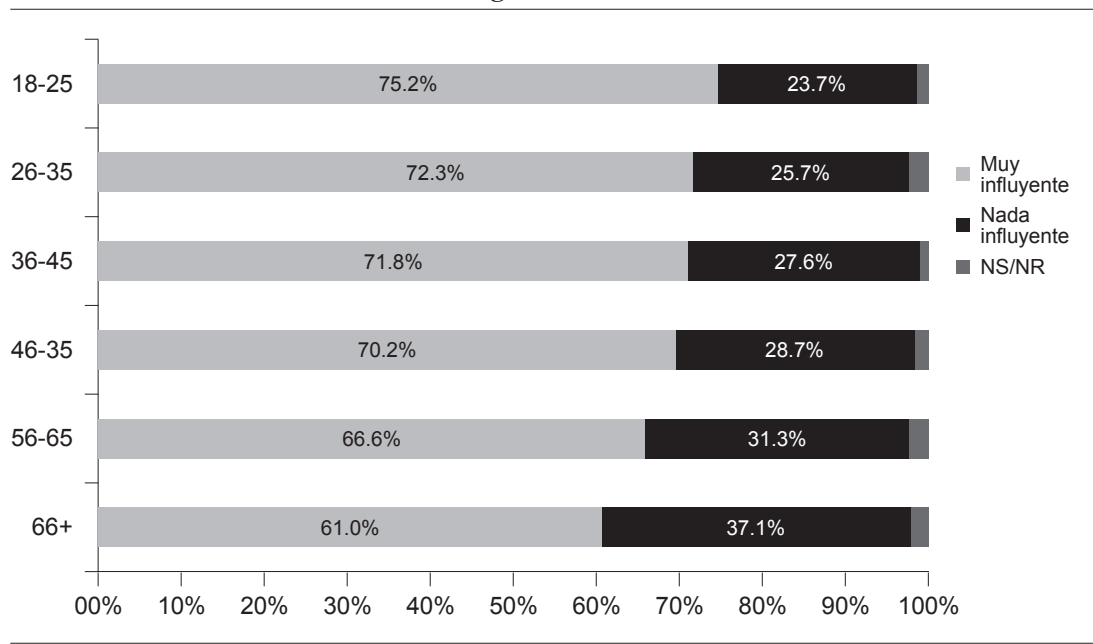

Fuente: Misión de Observación Electoral, MOE (2011)

cuentas activas. Existen casi once millones de cuentas de Facebook de usuarios entre los 13 y los 24 años en Colombia, y los usuarios que pueden votar, ubicados entre los 18 y 24 años de edad, son 7526720 . Población atractiva no solo para cualquier candidato que pensaría en movilizarlos hacia las urnas a partir de los 18 años, sino también porque contribuirían al alineamiento desde los partidos y desde las tendencias políticas que abonan el terreno para fortalecer la democracia. En el área metropolitana de Bucaramanga hay, según datos de owloo.com (2014), un aproximado de 608000 cuentas de Facebook, al interior de una población proyectada en su área metropolitana de 967224 habitantes, es decir un 63\% de las personas tienen cuenta en la red social, mientras que la media nacional es del $43 \%$ con respecto a la población total de habitantes en Colombia. No obstante, estos altos porcentajes, es necesario hacer la salvedad de que existe también en los jóvenes la tendencia de tener más de una identidad en esta red social.

Pese a que el uso de las redes sociales puede tener características que la pueden hacer mayormente participativa en política, de manera 


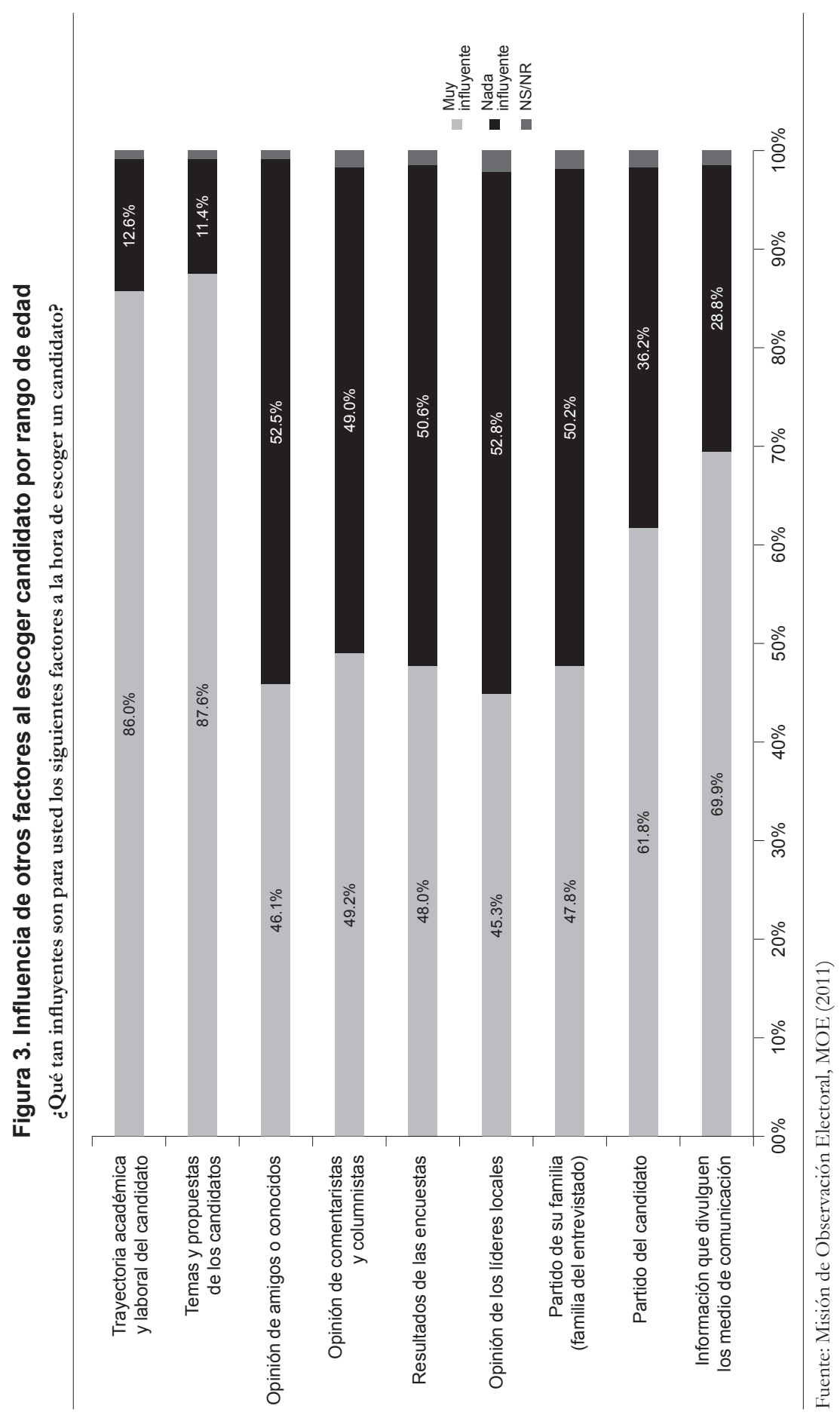


continuada y sin intermediarios, también hay que andarse con cuidado por el nivel de mentira o desinformación originada en la permisividad del anonimato, por ello existe la singularidad, que en palabras de Albero, (2010) "puede democratizar el debate público, pero también es verdad que el debate puede resultar superficial, sin respeto por las pruebas o los argumentos lógicos y terminar convirtiéndose en un diálogo incoherente entre ignorantes” (p. 18).

La diferencia entre las campañas políticas en el siglo Xx y el XXI tiene en el uso del concepto En Línea toda una revolución. Según Jhonson (2011), lo que en un comienzo fue el uso de correos electrónicos y portales ha cambiado. Ahora se necesita todo un equipo dedicado al trabajo En Línea, que esté metido en toda la campaña y que sea capaz de encontrar la participación ciudadana y mantenerla allí. Igualmente, aunque la televisión sigue siendo un medio importante, los lugares virtuales para dejar videos y compartirlos en redes como Facebook y Twitter son muy relevantes, ya que tienen menos límites y son más baratos, y las campañas son mucho más veloces, tanto que requieren estar alertas todo el tiempo: "The campaign now sleeps only when the elección is over" (p. 9).

\section{El tema del momento: entre la opinión, el compartir y la movilización}

Algunos estudios como el de Arriagada y Scherman (citados por Scherman et al., 2011) dan cuenta de que, para el caso de redes sociales, la participación política es un factor útil para predecir alineamientos o comportamientos, e incluso para fomentarla positivamente, exponiendo además que sería interesante estudiar cómo se conducen efectivamente las prácticas deliberativas y si tienen incidencia en la votación.

Ya se había mencionado que una de las regularidades de la participación electoral en los jóvenes es la de responder más a la atmósfera del momento, y que el uso de redes sociales se presta para la extensión de informaciones compartidas, sin embargo, el proceso de conocer si el uso de Internet promueve una nueva cultura política basada en contextos endémicos o si podría ser un medio novedoso, llamativo 
y con resultados esperados está por descubrirse. En el segundo caso, bien podría pensarse en una alternativa para el mercadeo de candidatos y de partidos.

En una reciente conferencia sobre Marketing Político llevada a cabo en Bogotá, la conferencista venezolana Sheila Dallmeier (2014) —coordinadora de la movilización de la campaña del candidato venezolano Enrique Capriles-comentó un hecho ocurrido en la campaña por la alcaldía de Bogotá en 2011 de Jaime Castro, exalcalde en 1992. Castro compitió en las elecciones del año 2011 contra Gustavo Petro, Enrique Peñaloza, Gina Parody y ocho candidatos más; en la campaña, un especialista en mercadeo político en redes sociales aconsejó adelantar una estratégica ofensiva virtual y, luego de hacer un filtro de posibles votantes, entre quienes se encontraban los que opinaban y participaban favorablemente hacia el candidato, decidieron invitar a 1000 simpatizantes a su sede política. La sorpresa que se llevaron fue enorme cuando solamente una persona acudió a la cita. Posteriormente, el candidato fue el último de los nueve, obteniendo tan solo 10509 votos (0,46\%), comparado con el ganador Gustavo Petro que logró $721508(32,16 \%)$.

Precisamente, desde el mercadeo político, que tiene la perspectiva del votante como un cliente, y bajo los conceptos de los productos del partido, sus metas y su mercadeo. Less-Marhment (2009) indica que dentro de las metas podría estar el cambio de la agenda del debate político, es decir, centrarlo en otras cosas y obtener nuevos segmentos en el mercado. Sin embargo, también una de las metas es aumentar la afiliación o la participación en su organización o partido político y garantizar el éxito electoral a largo plazo y no solo para una elección, caso que habría que estudiar dada la volatilidad de las redes sociales como medio.

En cuanto al ejercicio del poder en la red, Castells (2009) señalaba su funcionamiento variable en el tiempo, de acuerdo con las variaciones de las plataformas, la aparición de otras formas de funcionamiento y también a la capacidad de los usuarios como actores sociales para modificar su funcionalidad según sus propios intereses. "La sociedad 
red global es una estructura dinámica, altamente maleable a las fuerzas sociales, la cultura, la política y las estrategias económicas” (p. 58).

Señala también que el poder de influenciar en los comportamientos de otras personas requiere de dos mecanismos básicos. Por una parte, el de programar o reprogramar las redes según diferentes objetivos y, por otra parte, conectar otras redes de manera estratégica, tal como sucede con los medios de comunicación tradicionales que generan contenido en la red y de blogs, Twitter con contenidos favorables o llamativos hacia el candidato, con el propósito de evitar la competencia de otras redes (p. 76).

En cuanto a la participación política, algunas investigaciones, (Brader, 2006, Westen, 2007) han encontrado que las personas tienen la tendencia a continuar creyendo en sus propias convicciones políticas, siendo motivadas por elementos como el entusiasmo y el miedo, desde el apoyo al candidato de preferencia o al miedo de votar por el contrario. Sería muy interesante observar estas dos motivaciones en las últimas elecciones presidenciales en Colombia para entender cuántas personas votaron por Juan Manuel Santos, porque tenían miedo al otro candidato y viceversa. Hacer surgir un miedo aún mayor a la simple animadversión natural hacia el candidato que no es de la preferencia, puede ayudar también a conseguir nuevos votantes, especialmente cuando son presentadas las posibles consecuencias negativas en el caso de la victoria de uno u otro.

Precisamente, Brader (Citado por Castells, 2009) encuentra en su investigación, "que no había oposición entre los asuntos emocionales y los racionales. Las emociones [...] confieren fuerza al argumento" (p. 210). De tal manera que los jóvenes podrían ser motivados en Facebook a votar por los candidatos que se adecuaran a sus sentimientos y emociones, más que por los que presenten mejores argumentos. Este factor puede ser muy importante para entender su comportamiento.

Las críticas hacia el Gobierno, hacia los partidos políticos y especialmente hacia el Congreso, que según las encuestas en Colombia es el 
organismo estatal con mayor desprestigio, sugieren que efectivamente podrían estar trasladándose hacia un aumento del voto en blanco, que en las elecciones del 9 de marzo de 2014 se presentó con un 6,16\% en el Senado y de un 6,56\% en la Cámara, habiendo oscilado históricamente entre el 3\% y el $5 \%$. Aunque se puede observar un ligero aumento, finalmente no fue tan significativo a pesar de las masivas campañas y adeptos declarados en las redes sociales, que según encuestas desde enero hasta abril de 2014, que lo situaban desde un 22\% hasta un $15 \%$, terminó finalmente siendo del $6 \%$ en las elecciones presidenciales del 25 de mayo (ElTiempo, "Los aciertos y desaciertos de las encuestas frente a las votaciones", 2014).

Igualmente, los altos niveles de abstención generalizados en la mayoría de las democracias del mundo, necesariamente no serían una consecuencia de la pérdida de legitimidad del sistema político. De acuerdo con Pérez (2006), no votar podría corresponder a una forma participativa de expresar el descontento y la inconformidad hacia el Gobierno, el sistema político o el Congreso. De hecho, en las recientes elecciones del 9 de marzo de 2014, además del incremento leve del voto en blanco, fue significativo el aumento de votos nulos, (1485567, correspondiente al 10,38\%) y aunque lo más probable es creer que este hecho obedece a la incapacidad o desconocimiento para marcar el tarjetón, también la Registraduría comentó que los votos marcados con insultos y reclamos fue muy grande.

Pérez (2000) señala que en Venezuela esta insatisfacción política hacia los partidos tradicionales, COPEI y AD, iniciada a finales de los años ochenta, modificó el panorama electoral, inicialmente con altos niveles de abstención como alternativa, encontrando que mientras la satisfacción con el sistema político disminuía, aumentaba la abstención, constituyendo en las elecciones de 1993 un 39,8 \% y posteriormente expresando la insatisfacción en el incentivo a votar por Chávez en 1998. Este porcentaje aumentó considerablemente, pues en las seis elecciones, que van desde 1958 a 1988, el promedio de abstención fue de apenas un $9,8 \%$, "pequeño en cualquier comparación internacional” (Molina, 1995, p. 161). 
Sin embargo, en Venezuela, ni las altas tasas de participación, ni el abstencionismo tienen un origen en variables sociodemográficas, "ni aparecen diferencias significativas entre jóvenes y adultos" (Molina, 1995, p 165). Allí valdría la pena establecer una mirada sobre la edad y los posibles cambios o permanencias en la participación electoral mediante el uso de nuevas tecnologías, nuevos medios digitales, pero sobre todo las redes sociales, toda vez que su uso político es creciente, dadas las condiciones actuales del país.

De hecho, en Venezuela la dificultad para distinguir entre la sub información y la desinformación es difícil para el mismo periodismo, dada la enorme polarización política existente. Díaz (2014) refiere que existiendo, por ejemplo, tres millones de cuentas de Twitter, la información compartida es sumamente antagónica y tiene que ver con el deseo que tiene la gente de replicar las noticias y los datos, según su deseo político o la misma información sacada de contexto para afirmar cosas que no suceden.

Por eso, son importantes los niveles de reputación de las personas políticamente muy activas en las redes sociales, que se van adquiriendo con el tiempo, pero que en todo caso requieren de un proceso de tamizado de los medios de comunicación que usan el contenido. "Incluso para periodistas que tienden a ser muy objetivos y equilibrados, se excusan en lo que dicen los blogueros: 'En Twitter se dice tal cosa' y en realidad es su propia selección la que está dañando la comunicación” (Díaz, 2014, p. 69).

Existen posibilidades de que las mismas características tecnológicas y de autonomía que representan estas redes para la participación política generen elementos para fortalecer o debilitar las democracias, ya que al existir la oportunidad para difundir información errónea, la incidencia en una identidad colectiva desinformada o manipulada es posible. La fiabilidad de la información es un factor sumamente importante. "En la medida en que la precisión de la información no se puede determinar, el potencial positivo de estas poderosas tecnologías puede ser mitigado si no es puesto en contra de sí mismo" (Rheingold, 2008, p. 237). 
Recientes estudios que relacionan los ciclos de vida con el ejercicio del voto en Estados Unidos, podrían abrir el panorama hacia otros elementos interventores en la decisión electoral, especialmente la presión social que, estimulada mediante mensajes, puede servir poderosamente para aumentar la participación política, lo que evidencia que el factor edad es importante para establecer respuestas diferentes entre grupos de individuos. Panagopoulos y Abrajano (2014) encuentran que, a medida de que se aumenta en edad, la motivación a votar se incrementa al tener como variable la presión social como parte de un deber cívico. Su hipótesis presupone que los individuos más jóvenes tienden a ser más individualistas y con menos intereses en servir como ejemplos de valores democráticos, mientras que las personas de edad más avanzada tienden a pensar más en niveles colectivos y beneficiosos para la sociedad. La relación entre el deber de las personas mayores de servir como ejemplo para las más jóvenes es un factor motivacional y estimulante para votar, como si se tratara de una cierta norma social en la cual se educa mediante el ejemplo.

Los investigadores siempre tuvieron la duda de si las diferencias eran atribuibles a la edad o al factor generacional, al reconocer que, al igual que otras investigaciones de cohortes (Keeter, Zukin, \& Jenkins, 2002), empíricamente bajo factores estadísticos, no podrían demostrar el segundo factor. Precisamente, bajo factores de supervisión de comportamiento y vigilancia pública, para el caso de las redes sociales, valdría la pena preguntarse si las interacciones suscitadas en los muros podrían llevar, de alguna manera, a comportamientos del tipo presión social desde el punto de vista del deber ciudadano como modelo. Igualmente, sería interesante mirar si existe también una presión social ejercida por grupos generacionales diferentes u otros que pertenecen a la misma generación.

También podría ser un factor interventor para el análisis del uso de redes sociales en la participación política de los jóvenes, y teniendo en cuenta que se ha venido constituyendo en uno de los espacios más importantes de socialización, aquel que tiene que ver con la auto percepción y la imagen que quieren mostrar y que se inicia, 
especialmente en Facebook a edades muy tempranas de la adolescencia (13-15 años). Valdría la pena analizar si, a medida que aumenta la edad, también tienen la necesidad de construirse como sujetos políticos participativos con una auto imagen o una sobre exposición que busca una mayor valoración de parte de sus pares. Algunas de las características de los adolescentes colombianos y la necesidad de mostrarse como sujetos activos originales están siendo estudiados (Almansa-Martínez, Fonseca \& Castillo-Esparcia, 2013), e igualmente sería interesante mirar si estas características sobre filiación política e identificación partidista, que hacen parte de la información en la portada del Facebook, es conservada luego de cumplir los 18 años y pueden ejercer su derecho al voto.

Recientes estudios (Bakshy, Park \& Marlow, 2012) sobre la difusión de información usando redes sociales, exponen que no es cierto que cuanto más sea compartida tiende a ser más novedosa y efectiva, toda vez que hay una diferencia entre la fuerza de los lazos que los pares o "amigos" en Facebook tienen entre sí, y el contenido de la información diferente que comparten, de tal manera que si bien es cierto que los amigos más cercanos comparten más información, la información novedosa se difunde más entre amigos que tienen vínculos más lejanos entre sí. A menudo, los amigos que se relacionan menos tienen más posibilidades de compartir información novedosa y quienes comparten información más a menudo, generalmente tienen las mismas fuentes y recursos para compartir. Es decir, es más probable que las piezas de información fluyan más por medio de lazos débiles.

La característica de que exista un mayor incentivo a participar políticamente entre personas menos conocidas fue sugerida también por Gil de Zúñiga y Valenzuela (2011), se encontró que las redes sociales son un medio ideal para hacerlo, ya que es todo un centro de múltiples informaciones y contenidos de compromiso cívico, que se relacionan positiva y similarmente, si se comparan tanto en las redes Fuera de Línea como en las redes En Línea.

Otro elemento para tener en cuenta fue señalado por Gergen (2008), expresó que al existir una interacción diferente entre los grupos que 
participan políticamente cara a cara y los que participan en redes sociales, es posible distinguir dos grupos monádicos, diferenciados en el carácter de aquellos que tienen la tendencia a la afirmación circular de sus propias convicciones, mientras que:

$[\ldots]$ los que están fuera del grupo son vistos con indiferencia o desprecio. En efecto, los flujos de comunicación política esenciales para las democracias viables son interrumpidos. El diálogo y la comunicación entre los grupos da paso a un monólogo al interior de los grupos (p. 303).

Igualmente, es posible encontrar un mayor extremismo en la participación política nacional que en la internacional.

Si es posible encontrar que la información compartida entre amigos con lazos más cercanos es mayoritariamente redundante y predecible porque mantienen contenidos parecidos, y que la propagada entre quienes tienen lazos más débiles es más entrópica, siendo su contenido más interesante y novedoso, igualmente se podría trasladar el concepto de comunicación fática de Jakobson (citado por Fiske, 1985) según el cual, en las relaciones sociales es necesario esgrimir actos de comunicación, que en el caso de Facebook bien podría ser el mismo compartir o el like con el propósito de que el ego y la presencia sea notada, logrando "mantener o reforzar una relación ya existente" (p. 9), mientras que no hacerlo puede debilitar los lazos que se presumen fuertes. Entonces, podría ser que el contenido político compartido entre pares con lazos fuertes quizá se deba a una actitud de comportamiento y no necesariamente al propósito de informar, es decir, la comunicación fática sería redundante y no entrópica. Esta podría ser una explicación para que desde el Márketing político se sigan generando contenidos que finalmente serán compartidos por cortesía o por la visibilidad e impulso del ego y no por una convicción real de lograr mover hacia el alineamiento político.

Otros estudios estadísticos interesantes mantienen centrada la atención en cómo se propaga la información en Facebook, cuándo son usados contenidos que invitan a la participación. Adamic, Lento y 
Fiore (2012) encontraron que un meme $e^{2}$ muy popular que exhortaba a los amigos de la red a contestar la pregunta ¿cómo me habías conocido? con el objeto de responder, dejarlo en el muro y compartirlo, sirvió para establecer que la mayoría de personas de su análisis se había conocido por medio del colegio y que sus lazos eran incluso más vinculantes que las propias redes familiares o que los efectos de género al ser compartido. Este factor de compartir usando memes para expresarse políticamente está siendo usado en Colombia para polarizar los alineamientos de los jóvenes, y serían muy interesantes los estudios a este respecto.

Finalmente, otro factor interventor sería el señalado por Dumortier (2009), que explica que la amplia difusión de la información en Facebook, por medio de amigos que se encuentran en círculos distantes, con una enorme cantidad de gente que no se conoce, está alejada de la lógica natural en la que los seres humanos transmiten información. Por ejemplo, si es de manera oral, requiere de una expectativa racional derivada del interés de conocer a la persona referida. Por ello, puede ocurrir que la información política presentada y compartida se encuentre en contextos dispares y, aunque es posible que los usuarios se sientan a gusto en diferentes contextos, e incluso lleguen a adoptar diferentes personalidades, para la participación política se requiere construir patrones que contribuyan al alineamiento hacia tendencias, ojalá expresadas en los partidos políticos.

En otras palabras, el mercadeo político no debería brindar la posibilidad de compartir la información sin tener en cuenta contextos contradictorios. La manera de mercadear las ideas y actividades políticas en Facebook, pareciera ser la de poner información a circular, y lograr altos niveles de mensajes compartidos. Valdría la pena preguntarse ¿dentro de qué contexto un joven votante ve información política y decide opinar o compartirla?, ¿cuando está en la cafetería

\footnotetext{
2 Un meme es conocido en Internet como una representación simbólica, generalmente gráfica, que se transmite rápidamente, que tiene aspectos culturales compartidos y que representa ideas que van desde lo humorístico hasta mensajes profundos. La característica principal del meme es que aquello que representa es aceptado y en consecuencia compartido viralmente.
} 
de la universidad, caminando en un centro comercial o en la comodidad de su casa? Habría que averiguar mucho más sobre este tema.

El punto anterior lleva hacia el concepto de la visibilidad en Facebook, que es una cosa diferente a la participación. Si las audiencias son activas, de nada serviría la sola presencia sin participación. Sería una presencia paradójica, sería una presencia ausente de la red, que no representa muchos réditos en el mercadeo político, especialmente desde los jóvenes. Quizá, debido a ello, es que las experiencias han sido menos exitosas, por ejemplo, en España, donde solo el 20,28\% de los 350 diputados tiene presencia en la red, pero solamente el 9,71\% (34 perfiles) es abierto. No obstante, la estrategia de comunicación es unidireccional, político-ciudadano (López \& García, 2011). Precisamente, algunas experiencias exitosas de movilización política no se quedan en el fallecimiento en el mundo real de la motivación virtual. Las sentencias de quienes afirman que lo que sucede en el mundo virtual nace y muere en él, se equivocan cuando se logra pasar de la interacción a la acción real, como en las recientes movilizaciones de la llamada Primavera Árabe, las marchas de Un Millón de Voces contra las Farc o las marchas estudiantiles chilenas. En palabras de Cabalín (2014), se trata de una mezcla constante entre redes sociales y calle, combinando la información compartida virtualmente con el trabajo en varios lugares de diferentes ciudades y la ejecución activa de labores singulares y novedosas para los jóvenes.

\section{Conclusión}

Se puede decir que el factor edad es socialmente relevante e incidente en los resultados electorales del país y de Santander, cuyo potencial sería de 173226 jóvenes entre los 18 y 24 años y, sin embargo, es notorio por su baja participación real en las urnas, (alrededor de un $10 \%$ ), es decir, aproximadamente 7333 jóvenes, sobre una participación de aproximadamente 733000 votantes en Santander en las últimas elecciones de Senado y Cámara del $2014,{ }^{3}$ sin embargo, se

\footnotetext{
3 Estas cifras son especulativas ya que no han sido publicados datos oficiales del año 2014, pero están basadas en la abstención histórica y en declaraciones del Registrador Carlos Ariel Sánchez.
} 
cree que se conserva una característica especial de alta participación política en redes sociales.

En cuanto al factor edad como variable de la participación electoral en Colombia, podrían considerarse tres aspectos desde los enfoques de la Decisión Racional y el enfoque socio-psicológico que aumentan en el tiempo junto a una mayor intervención, las diferencias generacionales que corresponden a diferentes contextos, la consecución de recursos económicos y el nivel de aprendizaje y conocimiento, que también aumenta con la edad.

En cuanto a la influencia de la participación política en la participación electoral, desde las informaciones compartidas en las redes sociales, Facebook y Twitter, podrían hacerse distinciones entre la desinformación, la sub información y los contenidos políticos expuestos por los medios tradicionales, que pueden motivar la elección racional.

Desde los factores incidentes, tanto en la participación política, como en el ejercicio real del voto en urna, son aspectos interventores en las redes sociales:

1. El acceso y uso de nuevas tecnologías (redes sociales) en los jóvenes como variable que incide en la participación política y electoral.

2. La distinción entre desinformación, sub información, información de medios tradicionales que usan redes sociales e información oficial de candidatos o campañas, con el propósito de reconocer la fiabilidad y la verosimilitud.

3. La influencia de la reputación de los usuarios de las redes, bien sean periodistas, intelectuales o ciudadanos del común, en el incremento de la participación política.

4. El seguimiento individual de posibles votantes jóvenes como factor desde el Marketing Político. 
5. La participación política de usuarios jóvenes en las redes sociales cuando generan y comparten nuevo contenido, o cuando generan prácticas deliberativas alrededor de temas políticos, teniendo en cuenta si dichas prácticas son referencias circulares de sus propias convicciones o apuntan a un convencimiento de otros grupos.

6. La distinción de contextos endémicos para un análisis sincrónico o diacrónico de la participación política juvenil, teniendo en cuenta temas como cambios de agenda de candidatos y aparición de nuevos partidos o movimientos.

7. Los llamados a la abstención y al voto en blanco como participación política juvenil de protesta en las redes sociales.

8. El factor del estímulo tipo "presión social" como parte de un deber cívico hacia los jóvenes participantes en política en las redes sociales, de parte de otros grupos de individuos con diferencias generacionales.

9. El factor de "autoconstrucción" como sujetos políticos participativos de los jóvenes en las redes sociales, estableciendo si a medida que avanzan en edad existe relación con una mayor participación electoral.

10. El factor de "fortaleza" en los lazos entre los "amigos" o "pares" que comparten en las redes sociales, teniendo en cuenta si la información política es considerada o difundida en mayor o menor grado.

11. El factor de "actitud de comportamiento" como causa principal para compartir la información política en redes sociales por parte de jóvenes.

12. El uso de los memes como expresión participativa de los jóvenes en la política. 
13. El factor del contexto en el que se comparte la información en redes sociales y si influye en la valoración para luego ser extendida a otros pares.

Muchas preguntas quedan abiertas, además de la exploración de si los jóvenes podrían ser motivados a votar o motivarse a hacerlo al usar las redes sociales. También es una invitación a replantear el tema desde el tema del Marketing Político, en el cual los candidatos requieren de mayores estudios de efectividad, especialmente porque la sola participación en redes puede generar, además de ejercicios publicitarios como la recordación de candidatos, resultados esperados de participación real en urna. Probablemente deba responder a un seguimiento mayor del votante individual, además del posicionamiento de la imagen del candidato.

Aparentemente, el nivel de logro de una campaña debe pasar por una primera participación efectiva, como las mencionadas fiestas de Obama o la marcha llamada "Un millón de voces contra las Farc", cuyo slogan "No más Farc" logró canalizar por medio de Facebook una oleada de descontento enorme en la ciudadanía, que protestó por los secuestros y asesinatos del grupo armado ilegal. Según fuentes del grupo y de medios de comunicación como El Tiempo, lograron agrupar 250 mil usuarios en la red y, posteriormente, el 4 de febrero de 2008, sin tener datos precisos, se movilizaron seis millones de personas en 193 ciudades de Colombia y el mundo. Esta capacidad de convocatoria correspondió a comités de usuarios bien organizados y con labores específicas que contaron con un momento coyuntural.

La mejor manera de medir la incidencia debe tener dos momentos, el primero debe ser de interacción efectiva, más allá de un Me gusta o de un Retwitteo, que corresponda a la generación de nuevo contenido participativo, mediado por un monitoreo organizado, el siguiente es la medición de la participación real en urna.

También sería interesante mirar si el factor edad corresponde a una especie de abandono o exclusión de parte de élites políticas, ya que la estrategia evocada para la conquista de estos votos, además de la 
esperanzada inclusión en redes clientelares extendida en el país, no es precisamente la educación en democracia, que no ha tenido en Colombia una participación divulgada en las redes sociales como recurso mediático más allá de los simples mensajes de Marketing Político que usa las mismas palabras de "paz", "cambio", "inclusión” "prosperidad" "justicia" "reconciliación”, etc.

En Colombia, se ha visto en los últimos tiempos electorales un avance desinformativo que aún continúa con la polarización política y que, sin embargo, requiere de un reordenamiento desde sus propósitos y hacia sus fines. El proceso de filtrado es también un compromiso de los periodistas para actuar y compartir contenido en redes sociales, siendo un factor muy importante dada la inmediatez de la participación y el uso juvenil de millones de ciudadanos que ven en ellas una oportunidad de expresión. "Necesitamos pensar que la red es eso que nos sostiene cuando nos caemos, para los que quieran entender qué es lo que está pasando; lo que nos sostiene cuando se necesita asistencia" (Díaz, 2014).

\section{Referencias}

Adamic, L. A., Lento, T. M., \& Fiore, A. T. (2012). How You Met Me. Michigan: Association for the Advancement of Artificial Intelligence. Retrieved from http://www-personal.umich.edu/ ladamic/papers/ memes/howmet.pdf

Albero, M. (2010). Internet, jóvenes y participación civicopolítica. Barcelona: Editorial Octaedro.

Almansa-Martínez, A., Fonseca, O., \& Castillo-Esparcia, A. (2013). Social Networks and Young People. Comparative Study of Facebook between Colombia and Spain. Comunicar, 20(40), 127-135. doi:10.3916/ C40-2013-03-03

Álvarez, L., Granados, V., \& Hernández, N. (2013). Actitudes hacia la política en estudiantes universitarios, sus correlaciones con la edad, el estrato socioeconómico y el nivel educativo, y diferencias según el género en la ciudad de Bucaramanga (Colombia). Reflexión Politica, 15(29), 120-138. Anduiza, E., \& Bosch, A. (2004). Comportamiento Político y Electoral. Barcelona: Editorial Ariel. 
Anduiza, E., Cantijoch, M., Colombo, C., Gallego A., \& Salcedo, J. (2010). Los usos políticos de Internet en España. Revista Española de Investigaciones Sociológicas, 1(129), 133-146.

Bakshy, E., Park, M., \& Marlow, C. (2012). The Role of Social Networks in Information Diffusion. In Proceedings of the $21^{\text {st }}$ international conference on World Wide Web. doi:10.1145/2187836.2187907

Berganza, M., \& García M. (2005). El método científico aplicado a la investigación en Comunicación Mediática. En M. Berganza (Ed.), Investigar en Comunicación. Guía práctica de métodos y técnicas de investigación Social en Comunicación (pp. 19-42). Madrid: McGraw Hill.

Brader, T. (2006). Campaigning for Hearts and Minds, How Emotional Appeals in Political And Work. Chicago: University of Chicago Press.

Bravo, J. (17 de mayo, 2013). El voto joven en Panamá. Mundo electoral. Recuperado de http:/ / www.mundoelectoral.com/html/index.php?id=1057

Cabalin, C. (2014). Estudiantes conectados y movilizados: El uso de Facebook en las protestas estudiantiles en Chile. Comunicar 43(XXII), 25-33. Recuperado de http:/ /www.revistacomunicar.com/index.php?conte nido $=$ detalles\&numero $=43 \&$ articulo $=43-2014-02$

Castells, M. (octubre, 2000). Lección inaugural del programa de doctorado sobre la sociedad de la información y el conocimiento. En G. Ferraté (Rector). Barcelona: Universitá Oberta de Catalunya, . Recuperado de http://tecnologiaedu.us.es/nweb/htm/pdf/106.pdf

Castells, M. (2008). Afterword. En J. E. Katz. (Ed.), Handbook of Mobile Communication Studies (pp. 447-451). Cambridge: MIT Press.

Castells, M. (2009). Comunicación y Poder. Madrid: Alianza Editorial.

Clavijo, B \& Morera, L. (2010) Elecciones presidenciales en Colombia 2010. Reflexiones Liberales. Instituto de Ciencia Política Hernán Echavarría Olózoga. Recuperado de http:/ / www.icpcolombia.org/archivos/conceptos/Elecciones_presidenciales_Colombia_2010final.pdf

Dallmeier, S. (febrero, 2014). Seminario Internacional de Márketing Político. Universidad Central, Bogotá.

David, T. (2008). How Obama Really Did It. Technol Rev, 111(5), 78-83. Recuperado de http://cs12.cs.qc.cuny.edu/ waxman/8fn_002.pdf Díaz, L. (2014). Alianzas poderosas: Redes sociales y periodismo. En F, Posada (Ed.), Rastreando datos. VII Encuentro de periodismo de investigación. 
(pp. 65-82). Bogotá, Colombia: Fundación colombiana javeriana de artes gráficas.

Dumortier, F. (2009). Facebook y los riesgos de la descontextualización de la información. Revista de Internet, Derecho y Politica, 9, 32-44. Recuperado de http://journals.uoc.edu/index.php/idp/article/view/ n9_dumortier/n9_dumortier_esp

El Tiempo (26 de mayo, 2014). Los aciertos y desaciertos de las encuestas frente a las votaciones. ElTiempo. Recuperado de http:/ / www.eltiempo. com/politica/partidos-politicos/elecciones-2014-como-le-fue-a-lasencuestas/14036230

Gergen, K. (2008). Movil Comunication and the Transformation of the Democratic Process. En J. E. Katz. (Ed.), Handbook of Mobile Communication Studie (pp. 297-309). Cambridge: MIT Press.

Gil de Zúñiga, H., \& Valenzuela, S. (june, 2011). The Mediating Path to a Stronger Citizenship: Online and Offline Networks, Weak Ties, and Civic Engagement. Communication Research, 38, 397-421.

Johnson, D. W. (2011). Campaigning in the twenty-first century: ¿a whole new ballgame? New York: Routledge.

Keeter, S., Zukin, C., Andolina, M., \& Jenkins, K. (2002). The Civic and Political Health of the Nation: a Generational Portrait. Center for information and research on civic learning and engagement (CIRCLE). College Park, MD.: The Pew Charitable Trusts Report.

Lees-Marshment, J. (2009). Political marketing: principles and applications. New York: Routledge.

López, M., \& García, J. (2011). Marketing Político, Gobierno y redes sociales: ¿Difundir, informar, comunicar, relacionarse? In II Congreso Internacional Latina de Comunicación Social (pp. 1-32). Tenerife: Universidad de la Laguna. Recuperado de http:/ /www.ull.es/publicaciones/ latina/10SLCS/actas_2010/001MiguelTunez02.htm

Merdidean Group (2014). Radiografia de Twitter en Colombia. Recuperado de http://www.meridean.co/Estudios/internet/radiografia-de-twitteren-colombia

Misión de Observación Electoral, MOE. (2011). Encuesta: Percepción electoral de los votantes colombianos. Recuperado de http://moe.org.co/home/ $\mathrm{doc} /$ encuesta/encuestamoe.pdf 
Molina, J. (1995). Los venezolanos abandonan el hábito de votar. La abstención en las elecciones de 1993. Boletín electoral latinoamericano, XIII, IIDH/CAPEL, 13, 159-179.

Molina, J. (2014). Factores socio-psicológicos del voto. Material de lectura, Seminario de Comportamiento electoral. Facultad de Ciencias Jurídicas y Políticas. Maracaibo: Universidad del Zulia.

Owloo.com (2014). Estadísticas de Facebook en Colombia. Recuperado de http:/ / www.owloo.com/facebook-stats/colombia/

Panagopoulos, C., \& Abrajano, M. A. (2014). Life-cycle effects on social pressure to vote. Electoral Studies, 33, 115-122.

Pérez, C. (2000). Cambios en la participación electoral venezolana: 19982000. Cuestiones Políticas 28, 9-21.

Pérez, C. (2006). Enfoques teórico-metodológicos en el estudio de la participación electoral. Cuestiones políticas 37, 111-111.

Pérez, C. (2008). Curso sobre campañas electorales y análisis del comportamiento electoral. Material de lectura sobre comportamiento electoral. Decanato de investigación y posgrado. Maracaibo: Universidad Católica Cecilio Acosta.

Presidencia de la República de Colombia. Programa presidencial Colombia Joven (2004). Politica nacional de juventud 2012-2015. Recuperado de http:// planipolis.iiep.unesco.org/upload/Youth/Colombia/Colombia_Politica_nacional_juventud.pdf

Rheingold, H. (2008). Movil Media and Political Colective Action. En J. E. Katz. (Ed.), Handbook of Mobile Communication Studies. (pp. 225-239) Cambridge: MIT Press.

Rodríguez, R. (2013). La fuerza electoral de las personas mayores: Comportamiento de voto y estrategias para persuadir a un colectivo cada vez más decisivo en campañas. En K. Sanders (Ed.), Estudios de comunicación politica (pp. 47-77). Madrid: Tecnos.

Sánchez, C. A. (11 de diciembre, 2013). Foro sobre el papel del ciudadano en el fortalecimiento de la democracia. Noticias RPTV. Recuperado de http: / / www.noticiasrptv.com/ site/vernoticia.asp? $\mathrm{ac}=\mathrm{Solo} \% 20 \mathrm{el} \% 20$ 10\% $\% 20$ de $\% 201$ os $\% 20 j \%$ C3 $\%$ B3venes $\% 20$ votan $\% 20$ en $\% 20$ el $\% 20$ pa $\%$ C3\%ADs. $\% 20 \& W P L A C A=503$

Scherman, A., Arriagada, A., \& Valenzuela, S. (2011). ¿Hacia una nueva ciudadanía multifuncional? Uso de medios digitales, redes sociales online y participación política. Revista Latinoamericana de Opinión Pública, 2, (159-191). 
Aspectos interventores en La PARTicipación Política y electoral de Jóvenes / 81

Sojo, C. (2011). El Estado bajo escrutinio. Opinión pública, estatalidady desempeño gubernamental en América Latina. Santiago: CEPAL.

Westen, Drew. (2007). The Political Brain, The Role of Emotion in Deciding the Fate of the Nation. Nueva York: Public Affairs. 
